\title{
Surgical Outcomes of Long Fusion Using Dual Iliac Screws Bilaterally for Adult Spinal Deformities: The Effect on the Loosening Rate and Sacroiliac Joint Correction
}

\author{
Shigeto Ebata ${ }^{1)}$, Hiroki Oba ${ }^{2)}$, Tetsuro Ohba ${ }^{1)}$, Jun Takahashi $^{2)}$, Shota Ikegami ${ }^{2)}$, Kensuke Koyama ${ }^{1)}$, \\ Hiroyuki Kato ${ }^{2)}$ and Hirotaka Haro ${ }^{1)}$ \\ 1) Department of Orthopaedic Surgery, Graduate School of Medicine, University of Yamanashi, Yamanashi, Japan \\ 2) Department of Orthopaedic Surgery, Shinshu University School of Medicine, Matsumoto, Japan
}

\begin{abstract}
:
Introduction: We recently demonstrated that pelvic incidence (PI) decreases after long fusion using iliac screws (ISs) and plays a role in good sagittal balance postoperatively. By contrast, the IS loosening rate may cause reversion, increasing the PI and causing loss of sagittal balance. The aim of this study was to determine the effect of the number of ISs inserted into the iliac bone for long fusion to correct adult spinal deformities (ASDs) on the frequency of IS loosening, postoperative PI, and surgical outcomes.

Methods: We included data from 70 consecutive patients. Cases in which two ISs were inserted bilaterally comprised the dual IS group (Group D), whereas cases in which one IS was inserted bilaterally comprised the single IS group (Group S).

Results: IS loosening was observed in four patients in Group D (9\%) and 14 patients in Group S (61\%). Both early and one-year postoperative PI were significantly smaller in Group D $(P<0.001)$. The sagittal vertical axis (SVA) one-year postoperatively was significantly smaller in Group D $(P=0.003)$.

Conclusions: The loosening rate of dual ISs was as low as about one-seventh that of single ISs. Using dual ISs, postoperative PI can be kept small, possibly resulting in a smaller SVA.

Keywords:

adult spinal deformity, iliac screws loosening, dual iliac screws, spinopelvic parameter, sagittal balance, sacroiliac joint, pelvic anchor

Spine Surg Relat Res 2019; 3(3): 236-243

dx.doi.org/10.22603/ssrr.2018-0090
\end{abstract}

\section{Introduction}

It is now well established that spinal malalignment has an impact on pain and disability in adults ${ }^{1-3)}$. Long fusion from the sacrum to the thoracic spine is a common surgical treatment option to correct for sagittal imbalance ${ }^{4)}$. Although a high rate of loosening of iliac screws (ISs) used as pelvic anchors for long fusion has been reported, it has not been considered a great problem thus far ${ }^{5}$. This is because patient-specific values for pelvic incidence (PI) have been used as key parameters for determining the ideal lumbar lordosis (LL) as a target for corrective surgery ${ }^{6,7}$, but it is not well known whether PI itself changes by surgery.

In order to compensate for sagittal imbalance, mechanisms such as pelvic retrograde and knee flexion are effec- tive. When spinal kyphosis increases, a force bending the sacroiliac joint (SIJ) occurs. As a result, the SIJ may nutate beyond the physiological range ${ }^{8}$. Pelvic anchors are important in surgeries for adult spinal deformities (ASDs). However, there have been few reports on the importance of pelvic correction for compensating SIJ and the changes that occur in pelvic fixation.

PI decreases after long fusion using $\mathrm{ISs}^{8)}$; the smaller the PI after surgery, the better the sagittal balance that can be maintained $^{9}$. Loosening of ISs may cause reversion to increased $\mathrm{PI}^{9}$. The decrease in PI causes counternutation of $\mathrm{SIJ}^{10,11)}$. If the ISs, the only screws inserted on the caudal side of the SIJ, loosen, the SIJ may nutate again. Consequently, the PI may increase again. Loosening of the IS may not lead to corrective loss of LL; however, as a result of in-

Corresponding author: Hirotaka Haro, haro@yamanashi.ac.jp

Received: October 22, 2018, Accepted: November 26, 2018, Advance Publication: January 25, 2019

Copyright (C) 2019 The Japanese Society for Spine Surgery and Related Research 


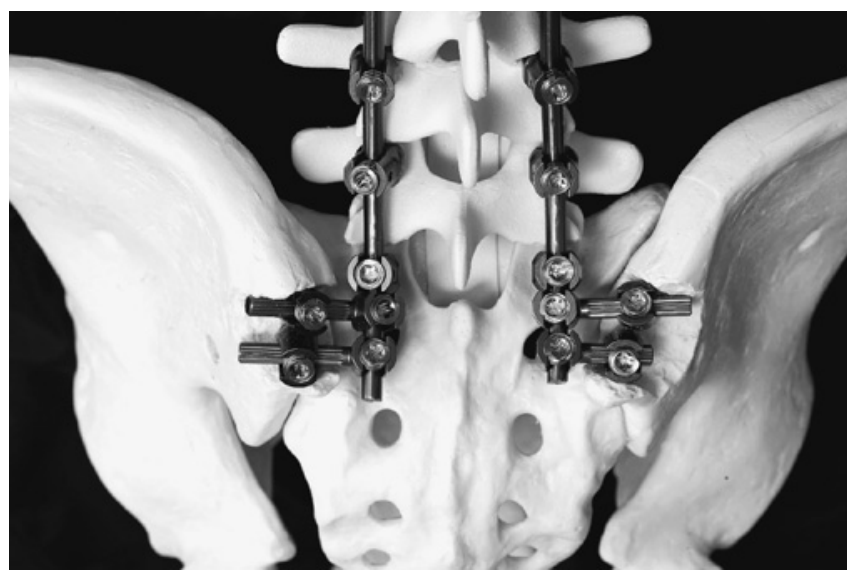

Figure 1. Photographs demonstrating the bone model. Dual ISs are bound to the S1 pedicle screw with a rod and a connector on each side. By pressing a curved rod into the three screw heads, it is possible to apply a correction force between the sacrum and the iliac. The size of the IS commonly used is $7.5 \mathrm{~mm}$, 50-70 mm long.

creasing PI, it may exacerbate sagittal imbalance.

We used single ISs, in which the ISs are inserted one by one bilaterally as pelvic anchors, or dual ISs, in which the ISs are also inserted bilaterally, but two on each side. However, to our knowledge, the clinical outcomes as a result of the number of inserted ISs have not yet been studied. By increasing the number of inserted ISs, it may be possible to correct the SIJ more strongly and the rate of loosening of ISs may decrease.

The purpose of the present study was to determine the effect of the number of screws inserted into the iliac bone in long fusion to treat ASDs on the frequency of IS loosening, postoperative PI, and surgical outcomes.

In order to determine these three outcomes, we performed a retrospective observational study on a cohort of 70 consecutive patients with ASDs who had undergone long fusion with either dual ISs or single ISs and had been followed up for more than one year.

\section{Materials and Methods}

This study was approved by the Clinical Research Ethics Committee. Informed consent was obtained from all the participants, and the study was conducted according to the Declaration of Helsinki.

\section{Patients}

In the present study, we included data from radiographs obtained from 70 consecutive patients in a full-standing position preoperatively, early postoperatively, and one year after surgery for correction of ASDs between January 2013 and January 2017. The inclusion criterion was a radiographic diagnosis of ASD defined by at least one of the following parameters: a C7 sagittal vertical axis (SVA) of $>50$ $\mathrm{mm}$, which is the distance between the $\mathrm{C} 7$ plumb line and the posterosuperior edge of $\mathrm{S} 1$, and a pelvic tilt (PT) of $>$ $30^{\circ}$, which is the orientation of the pelvis with respect to the femurs and the rest of the body. Patients with ASD underwent correction surgery with single ISs between January 2013 and April 2014 or with dual ISs between May 2014 and January 2017. They were excluded if they had ankylosing spondylitis or had undergone surgery without bilateral ISs. Basic demographic and surgical data (i.e., sex, age at surgery, number of fixed vertebrae, and type of procedure) were collected.

\section{Surgical procedure}

An anterior approach was selected to perform lateral interbody fusion (LIF) or posterior lumbar interbody fusion (PLIF) from the L1-2 or L2-3 to the level of the L4-5 disk in order to obtain an adequate coronal and sagittal spine alignment in patients with ASD. Then, the position of the patient was changed to a prone position. PLIF at the level of the L5-S1 disk was performed, and spinal kyphosis was corrected through cantilever force using bilateral S1 screws and bilateral ISs (Fig. 1). There was no case in which the PI screw was impossible to install in both single and double IS groups. In the case of loss of flexibility of spinal motion, Ponte osteotomy, pedicle subtraction osteotomy, or vertebral column osteotomy was added. To set the IS, a surrounder was directed to the line from the anterior superior iliac spine to the greater trochanter in a manner similar to that used in the ball-tip method ${ }^{12)}$. When inserting the second IS, another surrounder was inserted as close as possible and parallel to the primary surrounder. A $7.5 \mathrm{~mm}$ screw, $70 \mathrm{~mm}$ long, is usually placed, whereas 7.5-8.5 $\mathrm{mm}$ screws, $50-80 \mathrm{~mm}$ long, are sometimes used depending on the patients. Rods of adequate length, extending from the cranial to the caudal surgical level, were prepared. Lateral connectors bound the ISs to the rods, which were concatenated with S1 pedicle screws. Spinopelvic deformities were corrected using a cantilever force technique with the pelvis retroverted, raising the pelvis to an optimal alignment. Allogenic and local autogenous bone grafts were used. BMP was not used (Fig. 2).

\section{Radiographic measurements}

Two of the authors, who are senior spinal surgeons, conducted all the radiographic measurements. Full-length X-ray images from patients in a freestanding posture with their fingers placed on their clavicles were obtained before and after corrective surgery, but before discharge from the hospital (defined as early postoperative) and one-year postoperatively. We measured the spinopelvic parameters on these images, including PI, sacral slope, PT, T12-S1 LL, SVA, global tilt $(\mathrm{GT})^{13)}$, which is the angle formed by the intersection of two lines (the first line is drawn from the center of $\mathrm{C}$ 7 to the center of the sacral endplate, and the second line is drawn from the center of the femoral heads to the center of the sacral endplate ${ }^{14}$ ), and the T1 pelvic angle (TPA) $)^{15}$, which is the angle between the line from the center of the femoral heads to the center of $\mathrm{S} 1$ and the line from the 
femoral head to the center of the T1 vertebra ${ }^{5,16}$. IS loosening was assessed using anteroposterior X-ray images obtained over the course of the follow-up. Screw loosening was defined as a radiolucent area ( $\geq 1 \mathrm{~mm}$ in circumference) around the screw, noted on a plain radiograph by two observers $^{17,18)}$. In case of ambiguity, computed tomography scans were also used to assess loosening ${ }^{5)}$.

\section{Clinical outcomes}

The postoperative baseline health status of the patients was evaluated (for lumbar-pain-related factors) using the Oswestry Disability Index (ODI $)^{19)}$, where $0 \%$ indicates no disability and $100 \%$ indicates extremely debilitating disability. We assessed the one-year postoperative outcomes.

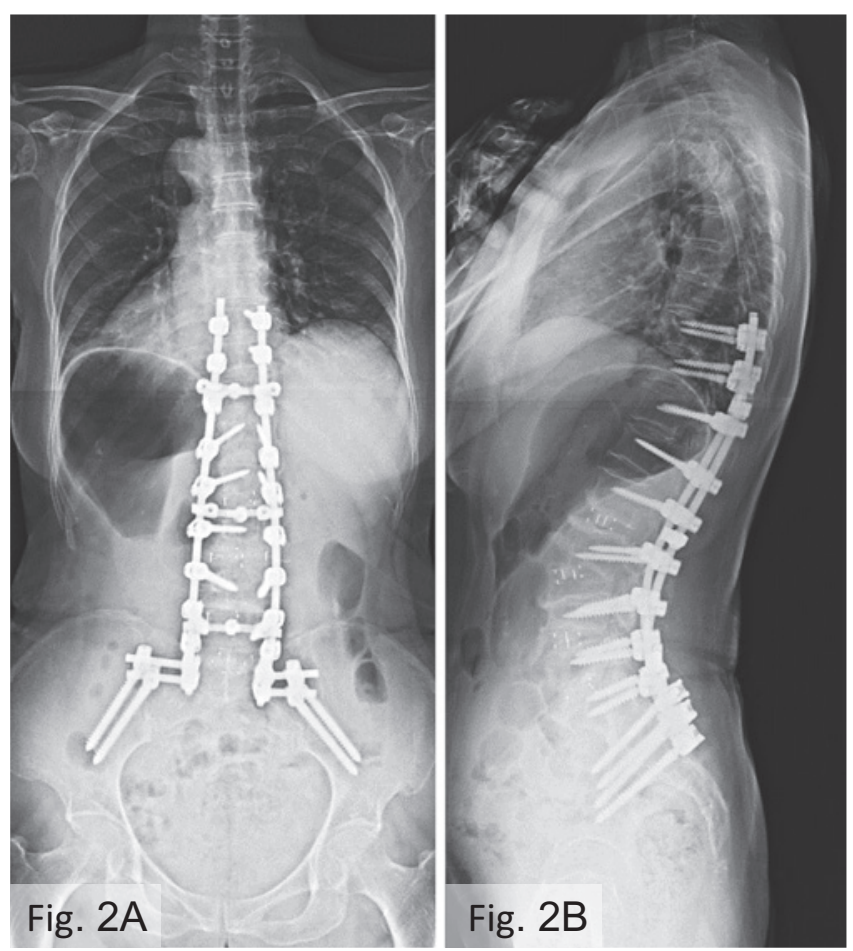

Figure 2. A: postoperative Anterior-Posterior view, B: postoperative lateral view.

Case Presentation. A 72-year-old female patient had ASD. She underwent surgery including LIF at L2-3, L3-4, and L4-5 disc levels and PLIF at L5-S1 and posterior corrective fusion from T10 to the ilium.

\section{Statistical analysis}

All data are reported as the mean (range). Patients were divided into two groups according to the number of ISs (Group D: with dual ISs, Group S: with single ISs). We compared the IS loosening and radiographic parameters between the two groups (longitudinally including preoperatively, early postoperatively, and one-year postoperatively). Interval or ratio scale values were compared using Welch's $t$-test, ordinal scale data were analyzed using the MannWhitney $U$ test, and nominal scale data were compared using Fisher's exact test. All statistical calculations were performed using the $\mathrm{R}$ statistical package (version 3.4.3; http:// www.r-project.org) and GraphPad Prism (version 6.0; GraphPad Software, La Jolla, CA, USA). In all analyses, a $P$-value of $<0.05$ was considered statistically significant.

\section{Results}

\section{Patient population}

We included data from the 70 eligible patients ( 8 females, 62 males) with a mean age of 70.6 (range: 50-80) years, and the mean number of fixed vertebrae was 9.8 (range: 5-15) vertebral bodies. The baseline patient characteristics in Groups D and S are summarized in Table 1. The mean preoperative, early postoperative, and one-year postoperative spinopelvic variables are summarized in Table 2. There was no significant difference in the preoperative spinopelvic parameters between the two groups.

\section{Comparison of the IS loosening rate between patients in Groups D and S (Fig. 3)}

When evaluated one year after surgery, the overall IS loosening rate was $25.7 \%$. The IS loosening rate was $8.5 \%$ in Group D and $60.9 \%$ in Group S. In Group D, the loosening rate of the IS was lower than that in Group $\mathrm{S}(P<$ $0.001)$. The displacement of the IS was $11 \%$ in Group $S$, but $0.5 \%$ in Group D.

\section{Comparison of changes in PI between patients in Groups $D$ and $S$ (Fig. 4)}

The PI values of patients in Groups D and S were, re-

Table 1. Baseline Patient Characteristics and Surgical Data in BIS (Bilateral iliac screw) s-D (dual) and BISs-S (single) Groups.

\begin{tabular}{lccc}
\hline \multicolumn{1}{c}{ Variable } & BISs-D $(n=47)$ & BISs-S $(n=23)$ & $P$ \\
\hline Age $(\mathrm{y})$ & $71.0 \pm 7.1$ & $69.8 \pm 7.3$ & 0.531 \\
Female (cases) & $43(91 \%)$ & $19(83 \%)$ & 0.279 \\
Parkinson's disease & 6 & 4 & 0.604 \\
Number of fixed vertebrae $(n)$ & $9.9 \pm 2.3$ & $9.5 \pm 2.7$ & 0.500 \\
Bleeding volume (g) & $989.1 \pm 730.7$ & $1,216 \pm 1,040.9$ & 0.293 \\
Operation time (min) & $513.6 \pm 94.1$ & $457.8 \pm 70.7$ & $0.014 *$ \\
\hline
\end{tabular}

Interval and ratio values are represented as the mean \pm standard deviation. 
Table 2. Baseline Spinopelvic Variables in BISs-D and BISs-S Groups.

\begin{tabular}{lccc}
\hline \multicolumn{1}{c}{ Variable } & BISs-D $(n=47)$ & BISs-S $(n=23)$ & $P$ \\
\hline SVA $(\mathrm{mm})$ & $108.0 \pm 65.4$ & $117.8 \pm 73.9$ & 0.575 \\
GT $\left(^{\circ}\right)$ & $48.8 \pm 16.8$ & $53.0 \pm 17.3$ & 0.337 \\
TPA $\left(^{\circ}\right)$ & $36.8 \pm 14.4$ & $41.5 \pm 14.2$ & 0.202 \\
PI $\left(^{\circ}\right)$ & $51.8 \pm 7.0$ & $53.6 \pm 7.8$ & 0.338 \\
LL $\left(^{\circ}\right)$ & $11.6 \pm 18.5$ & $12.0 \pm 17.1$ & 0.930 \\
PI-LL $\left(^{\circ}\right)$ & $40.2 \pm 17.7$ & $41.6 \pm 14.9$ & 0.748 \\
PT $\left(^{\circ}\right)$ & $34.7 \pm 11.6$ & $38.1 \pm 8.7$ & 0.821 \\
SS $\left(^{\circ}\right)$ & $16.3 \pm 13.0$ & $17.1 \pm 13.8$ & 0.821 \\
TK $\left({ }^{\circ}\right)$ & $25.1 \pm 18.4$ & $23.8 \pm 16.6$ & 0.764 \\
\hline
\end{tabular}

Interval and ratio values are represented as the mean \pm standard deviation. $* P<0.05$.

BIS: bilateral iliac screw

D: dual

S: single

PT: pelvic tilt

SS: sacral slope

LL: lumbar lordosis

PI: pelvic incidence

SVA: sagittal vertical axis

GT: global tilt

TPA: T1 pelvic angle

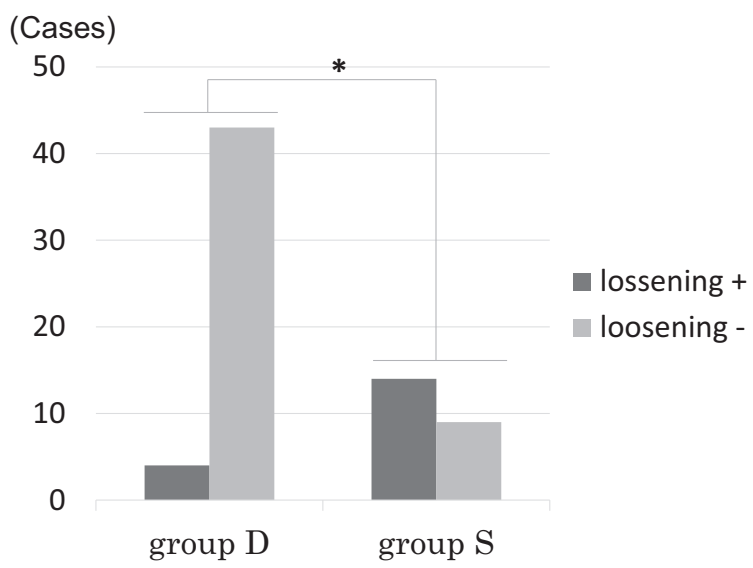

Figure 3. Loosening rate of ISs in Groups D and S. $* P<0.05$.

Group D: bilateral dual ISs

Group S: bilateral single ISs

Loosening+: with loosening of ISs

Loosening-: without loosening of ISs

PI: pelvic incidence

spectively, $51.8^{\circ}$ (range: $40.0-67.5^{\circ}$ ) and $53.6^{\circ}$ (range: 41.0 $\left.69.5^{\circ}\right)$ preoperatively, $44.9^{\circ}\left(31.0-64.5^{\circ}\right)$ and $54.6^{\circ}(44.0-$ $79.0^{\circ}$ ) early postoperatively, and $46.6^{\circ}$ (range: $33.5-66.5^{\circ}$ ) and $55.6^{\circ}$ (range: $44.0-80.5^{\circ}$ ) one-year postoperatively. The PI in the early postoperative and the one-year postoperative follow-up were both significantly lower for patients in Group D $(P<0.001)$. When comparing preoperative and one-year postoperative angles, the PI was found to be decreased in patients in Group D, but increased in patients in Group $\mathrm{S}(P<0.001, P=0.048)$.

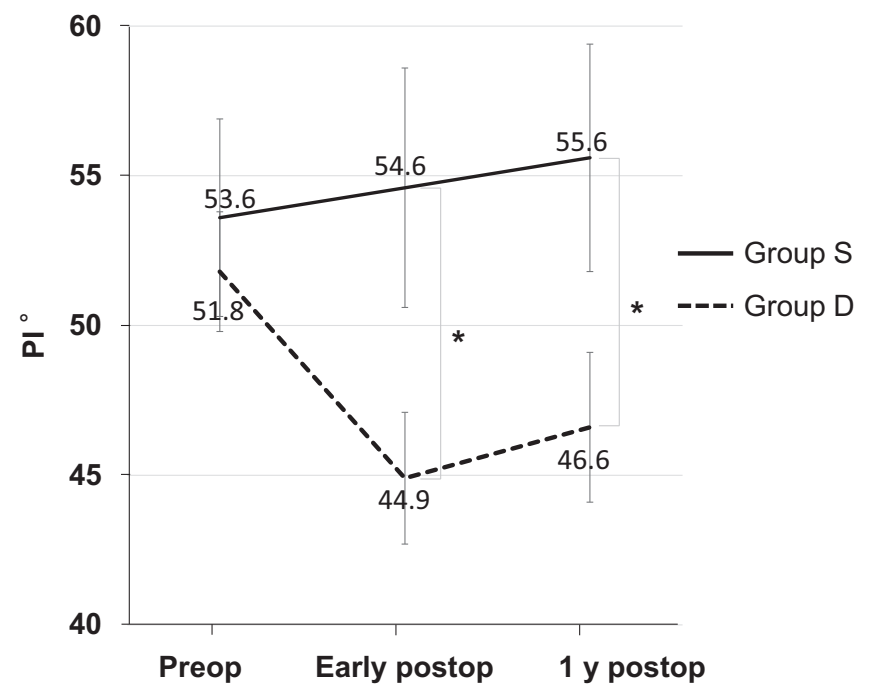

Figure 4. Postoperative change of PI in Groups D and S. $* P<0.05$.

Group D: bilateral dual ISs

Group S: bilateral single ISs

PI: pelvic incidence

\section{Comparison of spinopelvic parameters between patients in Groups $D$ and $S$ (Table 3)}

In the early postoperative period, GT, TPA, PI-LL, and PT were significantly smaller for patients in Group D than for those in Group S. The SVA of patients in Group S increased during the postoperative period. As a result, at oneyear postoperatively, GT, TPA, PI-LL, PT, and SVA were significantly smaller for patients in Group D than for those in Group S. The SVA differences between the two groups 
Table 3. Comparison of Spinopelvic Parameters between Patients with BISs-D and BISs-S.

\begin{tabular}{|c|c|c|c|}
\hline Variable & BISs-D (n=47) & BISs-S ( $n=23)$ & $P$ \\
\hline \multicolumn{4}{|c|}{ Early postop } \\
\hline SVA (mm) & $21.1 \pm 33.7$ & $38.4 \pm 45.2$ & 0.077 \\
\hline $\mathrm{GT}\left(^{\circ}\right)$ & $17.5 \pm 10.4$ & $32.4 \pm 10.4$ & $<0.001 *$ \\
\hline TPA $\left(^{\circ}\right)$ & $12.1 \pm 8.6$ & $25.1 \pm 8.6$ & $<0.001 *$ \\
\hline $\mathrm{LL}\left({ }^{\circ}\right)$ & $52.9 \pm 8.8$ & $44.0 \pm 10.0$ & $<0.001 *$ \\
\hline PI-LL $\left(^{\circ}\right)$ & $-8.0 \pm 10.4$ & $10.6 \pm 12.3$ & $<0.001 *$ \\
\hline $\mathrm{PT}\left({ }^{\circ}\right)$ & $15.2 \pm 7.0$ & $29.3 \pm 7.1$ & $<0.001 *$ \\
\hline $\mathrm{SS}\left({ }^{\circ}\right)$ & $30.6 \pm 8.1$ & $26.8 \pm 8.4$ & 0.073 \\
\hline \multicolumn{4}{|c|}{1 y postop } \\
\hline SVA (mm) & $29.0 \pm 34.1$ & $60.8 \pm 52.8$ & $0.003 *$ \\
\hline $\mathrm{GT}\left({ }^{\circ}\right)$ & $20.8 \pm 10.0$ & $36.4 \pm 11.3$ & $<0.001 *$ \\
\hline $\operatorname{TPA}\left(^{\circ}\right)$ & $16.4 \pm 7.5$ & $28.6 \pm 9.8$ & $<0.001 *$ \\
\hline $\operatorname{LL}\left({ }^{\circ}\right)$ & $51.1 \pm 9.4$ & $43.1 \pm 9.8$ & $0.001 *$ \\
\hline PI-LL $\left(^{\circ}\right)$ & $-4.5 \pm 10.4$ & $12.6 \pm 12.1$ & $<0.001 *$ \\
\hline $\mathrm{PT}\left({ }^{\circ}\right)$ & $19.5 \pm 6.9$ & $31.3 \pm 6.1$ & $<0.001 *$ \\
\hline $\mathrm{SS}\left({ }^{\circ}\right)$ & $28.6 \pm 7.6$ & $25.5 \pm 8.5$ & 0.125 \\
\hline \multicolumn{4}{|c|}{$\begin{array}{l}\text { Interval and ratio values are represented as the mean } \pm \text { standard deviation. } \\
* P<0.05 \text {. }\end{array}$} \\
\hline \multicolumn{4}{|c|}{ Early postop: early postoperatively } \\
\hline \multicolumn{4}{|c|}{1 y postop: one year postoperatively } \\
\hline \multicolumn{4}{|c|}{ BIS: bilateral iliac screw } \\
\hline \multicolumn{4}{|l|}{ D: dual } \\
\hline \multicolumn{4}{|l|}{ S: single } \\
\hline \multicolumn{4}{|c|}{ SVA: sagittal vertical axis } \\
\hline \multicolumn{4}{|l|}{ GT: global tilt } \\
\hline \multicolumn{4}{|c|}{ TPA: T1 pelvic angle } \\
\hline \multicolumn{4}{|c|}{ LL: lumbar lordosis } \\
\hline \multicolumn{4}{|c|}{ PI: pelvic incidence } \\
\hline \multicolumn{4}{|l|}{ PT: pelvic tilt } \\
\hline SS: sacral slope & & & \\
\hline
\end{tabular}

preoperatively, early postoperatively, and one-year postoperatively were $9.8 \mathrm{~mm}$ (95\% confidence interval $(95 \% \mathrm{CI})$ : -24.9 to $44 \mathrm{~mm}, P=0.575), 17.3 \mathrm{~mm}(95 \% \mathrm{CI}:-1.9$ to 36 $\mathrm{mm}, P=0.077)$, and $31.9 \mathrm{~mm}(95 \% \mathrm{CI}: 11.0-52.7 \mathrm{~mm}, P=$ $0.003)$, respectively.

\section{Comparison of clinical outcomes between patients in Groups $D$ and $S$ (Table 4)}

There was no significant difference in proximal junctional kyphosis and preoperative ODI. Postoperative ODI tended to be better for Group D than for Group S.

\section{Discussion}

Three things were made clear by the present study. First, patients in Group D had a significantly lower loosening rate of IS than patients in Group S. Second, patients in Group D had their PI decreased significantly by surgery compared to those in Group S. Third, one-year postoperatively, patients in Group D tended to have better sagittal balance and better clinical outcomes than those in Group S.

Patients in Group D had a significantly lower rate of IS loosening than those in Group S. Banno et al. retrospec- tively evaluated 72 patients with ASD who underwent long fusion with single ISs. They reported that IS loosening was found in 20 patients $(27.8 \%)$ and IS loosening appeared at 5.2 months on average ${ }^{5)}$. We similarly found a rate of loosening as high as $25.7 \%$ overall. However, limited to patients in Group D, the rate of loosening was as low as $8.5 \%$. PI decreased after ASD surgery with ISs, but a significant loss appeared with a year postoperatively. In addition, a significant loss of PI correction occurred within a year after surgery in patients with IS loosening different from the PI loss in those without screw loosening ${ }^{9}$. It has been shown in our recent study that $7.4 \%$ cases at one year and $11.1 \%$ cases at two years presented with IS loosening postoperatively in Group $\mathrm{D}^{20)}$. Thus, the bilateral IS system provides six points as pelvic anchors and may improve stability for long spinal and pelvic fusion.

There are at least two possible reasons for IS loosening. One is loosening as a result of being the most distal anchor of long fusion. The other is loosening because of the movement of the SIJ. If the instability remains in L5-S1, not only the pedicle screw of S1 (S1PS), but also the IS may be loosened. In seven patients $(38.9 \%)$ out of the 18 in whom the IS was loosened, S1PS was loosened; and in 11 patients 
Table 4. Comparison of PJK and Clinical Outcomes between Patients with BISs-D and BISs-S.

\begin{tabular}{cccc}
\hline Variable & BISs-D $(n=47)$ & BISs-S $(n=23)$ & $P$ \\
\hline Complication & $17(36 \%)$ & $9(39 \%)$ & 0.813 \\
PJK (cases) & $14.6 \pm 10.7$ & $8.7 \pm 9.7$ & 0.056 \\
PJA $\left(^{\circ}\right)$ & & & \\
Clinical outcome & $37.8 \pm 23.1$ & $45.1 \pm 20.7$ & 0.194 \\
Pre-ODI $(\%)$ & $23.5 \pm 19.1$ & $31.3 \pm 17.4$ & 0.099 \\
1 y ODI $(\%)$ & & & \\
\hline
\end{tabular}

Interval and ratio values are represented as the mean \pm standard deviation.

BIS: bilateral iliac screw

D: dual

S: single

Pre: preoperative

$1 \mathrm{y}$ : one year postoperative

PJK: proximal junctional kyphosis

PJA: proximal junctional angle

ODI: Oswestry Disability Index

(61.1\%), S1PS was not loosened. The movement of SIJ is suspected to be a cause of loosening for ISs.

Patients in Group D had a significantly decreased PI as a result of surgery compared with those in Group S. In order to compensate for sagittal imbalance, mechanisms such as pelvic retrograde and knee flexion are effective. When the spinal kyphosis further increases, a force bending the SIJ occurs. As a result, SIJ may nutate beyond the physiological range $^{9)}$. Pelvic anchors are important in ASD surgeries. The importance of pelvic correction for compensating SIJ and the changes that occur in pelvic fixation is clear. The range of movement of SIJs in adults is from $1^{\circ}$ to $4^{\circ}$ of rotation and 1 to $2 \mathrm{~mm}$ of translation ${ }^{21-24)}$. The complex movement of SIJs is called nutation and counternutation. PI increases because of nutation and decreases because of counternutation $^{10,11)}$. Legaye et al. reported the effect of age and sagittal imbalance on the variability of PI and concluded that they combined as key factors for an increase in $\mathrm{PI}^{25}$. Lee et al. studied postoperative PI changes in 29 patients with ASD who underwent long fusion. In a group without ISs, the PI increased by $9.4^{\circ}$ preoperatively until the final follow-up ${ }^{26}$. Perhaps this change was caused by repeated loads on the SIJ as the trunk is tilted forward. Cecchinato et al. evaluated the early postoperative PI in patients with ASD whose LIV was the ilium $(n=39)$ or S1 $(n=27)$ and found that PI increased in patients with an S1 LIV, whereas in patients whose LIV was the ilium, the PI decreased ${ }^{8}$. Oba et al. suggested that the SIJ of patients with ASD nutated beyond the physiological range because long-term loading may be corrected between the IS and S1PS ${ }^{9}$. PI increases when the SIJ is nutated, but it decreases when the SIJ is counternutated. In our previous study, PI decreased early after the correction surgery, but it increased during the first postoperative year because of IS loosening9). In our present study, patients in Group S showed no change in the PI, but those in Group D showed a significant decrease in the PI. Based on the iliac anchors created with two ISs, it might be possible to add a stronger correction force to the SIJ by pushing a curved rod into the S1PS screw head (Fig. 5).

One-year postoperatively, patients in Group D tended to have better sagittal balance and better clinical outcomes than those in Group S. The correction of LL itself was also slightly better in patients in Group D. Moreover, a large difference in PI-LL between the two groups was recognized. In patients in Group D, the PI could be reduced simultaneously with increasing LL, so PI-LL might have become smaller early postoperatively. Oba et al. reported that the smaller the PI early postoperatively, the less the reversion of SVA oneyear postoperatively. They suggested that, by reducing the PI by surgery using ISs, the one-year postoperative sagittal balance could be well maintained ${ }^{9}$. In our study, patients in Group D had a significantly smaller postoperative PI than what they had preoperatively. Compared with patients in Group S, those in Group D had a smaller SVA increase in the first postoperative period; and as a result, at one-year postoperatively, there was a significant difference in the SVA between the two groups. In addition, Oba et al. reported that loosening of the IS leads to a correction loss of $\mathrm{PI}^{9}$. Patients in Group D had a loosening rate about oneseventh that in those in Group S. This might also be the reason why, one-year postoperatively, the SVA increased less in patients in Group D than it did in patients in Group S. However, the proximal junctional angle in Group D was worse compared with that in Group S, although there was no significance difference (Table 4). The global sagittal balance, including SVA, GT, and TPA, was significantly better in Group D than in Group S (Table 3). Although we cannot specify the reason in the current study, the T1-UIV angle might be one of the factors. Future studies are needed to explain this question.

There are several potential limitations in our present study. First, data from a relatively small number of patients were included in the present study. Second, the study was retrospective. Third, it had a short follow-up period. Much 

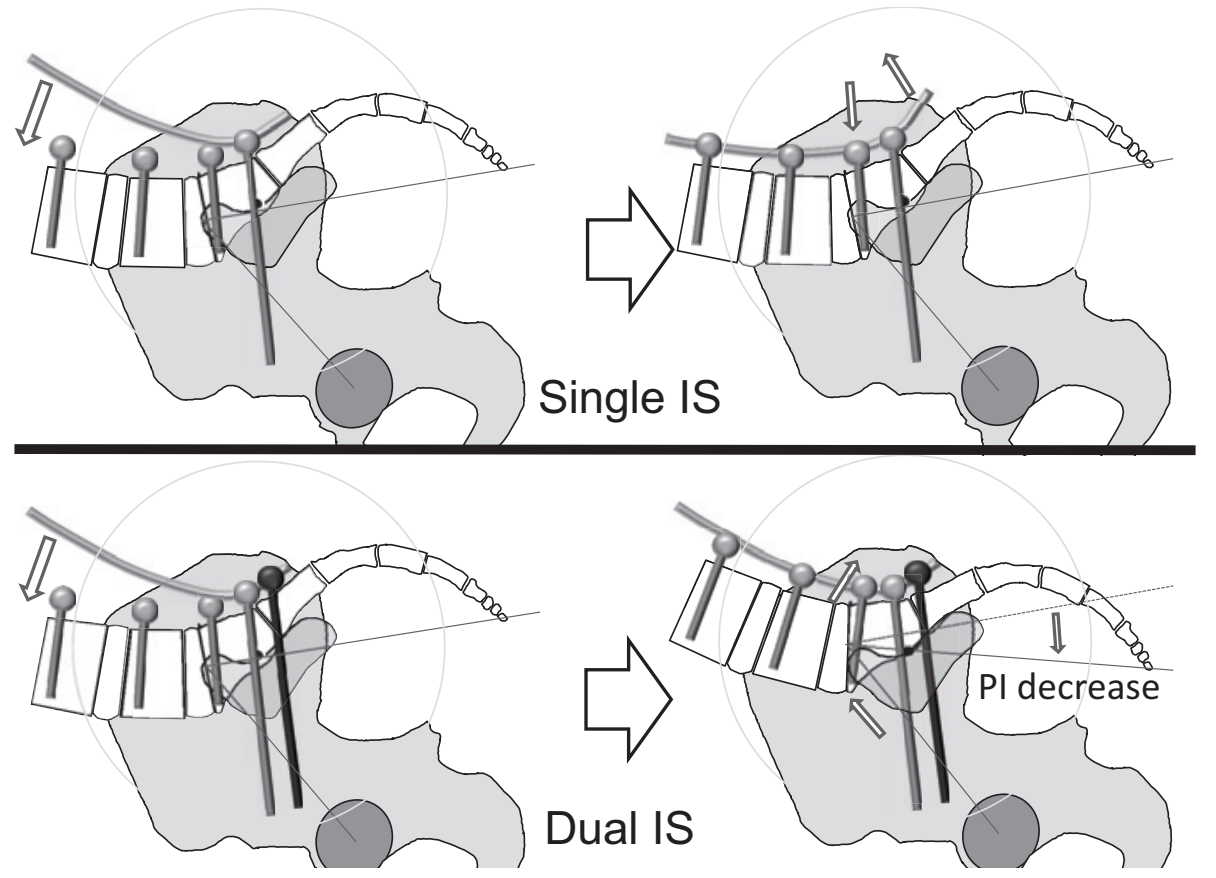

Figure 5. Difference in SIJ correction between using single ISs and using dual ISs.

SIJ: sacroiliac joint

IS: Iliac screws

PI: pelvic incidence

of the IS loosening was reported to occur early postoperatively $^{5}$; nevertheless, loosening should also be determined over a longer term. The loosening rate determined in this study is for only one year after surgery, and the long-term results are unknown. In addition, the selection of iliac number was determined the surgical period. Surgical skills to install ISs may be improved in Group D. Both the surgeons' experience for and the results of this retrospective study may be affected due to the time period (loosening rate, clinical results, and improvement of surgical correction skills). Bilateral IS systems may improve the stability for ASD surgeries and be able to add a stronger correction to the SIJ. However, there are problems regarding the learning curve to acquire the surgical skills of rod connection and screw installation, as well as the medical cost of screws. Further studies are needed to examine the cost-effectiveness.

Until now, IS loosening has not been often considered to be a great problem in long fusion. It is surprising that it has not attracted much attention to date. The results of our research suggest that, in order to maintain good sagittal balance, it is important that ISs do not loosen. There is a possibility that loosening may occur, even in patients in Group $\mathrm{D}$, if we observed a longer-term follow-up. This is because the load stress is constantly applied to the IS unless SIJ bones are fused. However, SIJs are cushioning joints and should not be fixed unless necessary. Nevertheless, if spinal fusion from the sacrum to the thoracic spine is performed, the PI may increase, unless the SIJs are strongly fixed. To avoid reexacerbation of sagittal imbalance because of PI increase, it may be necessary to consider not only a strong iliac anchor, but also bone grafting for SIJs. A limitation of this work is that a strong pelvic anchor known as a second sacral alar IS $^{27)}$ was not included in the present study.

\section{Conclusions}

The loosening rate of dual ISs was as low as about oneseventh that of single ISs. Using dual ISs, postoperative PI can be made smaller, possibly resulting in better sagittal balance than that obtained with single ISs. Evaluation of the presence or absence of longer-term loosening is warranted.

Conflicts of Interest: The authors declare that there are no relevant conflicts of interest.

Author Contributions: ES and HO participated in study design, data acquisition, and analysis of data. TO, JT, SI, $\mathrm{KK}$, and HK participated in data acquisition and analysis of data. $\mathrm{HH}$ participated in study design, analysis of data and prepared the manuscript. All authors have read, reviewed, and approved the article.

\section{References}

1. Schwab F, Farcy JP, Bridwell K, et al. A clinical impact classification of scoliosis in the adult. Spine. 2006;31(18):2109-14.

2. Glassman SD, Berven S, Bridwell $\mathrm{K}$, et al. Correlation of radiographic parameters and clinical symptoms in adult scoliosis. Spine. 2005;30(6):682-8.

3. Lafage V, Schwab F, Patel A, et al. Pelvic tilt and truncal inclination: two key radiographic parameters in the setting of adults with 
spinal deformity. Spine. 2009;34(17):E599-606.

4. Schwab F, Lafage V, Patel A, et al. Sagittal plane considerations and the pelvis in the adult patient. Spine. 2009;34(17):1828-33.

5. Banno T, Hasegawa T, Yamato Y, et al. Prevalence and risk factors of iliac screw loosening after adult spinal deformity surgery. Spine. 2017;42(17):E1024-30.

6. Yamato Y, Hasegawa T, Kobayashi S, et al. Calculation of the target lumbar lordosis angle for restoring an optimal pelvic tilt in elderly patients with adult spinal deformity. Spine. 2016;41(4):E 211-7.

7. Schwab F, Patel A, Ungar B, et al. Adult spinal deformitypostoperative standing imbalance: how much can you tolerate? An overview of key parameters in assessing alignment and planning corrective surgery. Spine. 2010;35(25):2224-31.

8. Cecchinato R, Redaelli A, Martini C, et al. Long fusions to S1 with or without pelvic fixation can induce relevant acute variations in pelvic incidence: a retrospective cohort study of adult spine deformity surgery. Eur Spine J. 2017;26(4):436-41.

9. Oba H, Ebata S, Takahashi J, et al. Loss of pelvic incidence correction after long fusion using iliac screws for adult spinal deformity: cause and effect on clinical outcome. Spine. 2018. Jul 2. doi: 10.1097/BRS.0000000000002775. Epub ahead of print

10. Egund N, Olsson TH, Schmid H, et al. Movements in the sacroiliac joints demonstrated with roentgen stereophotogrammetry. Acta Radiol Diagn (Stockh). 1978;19(5):833-46.

11. Alderink GJ. The sacroiliac joint: review of anatomy, mechanics, and function. J Orthop Sports Phys Ther. 1991;13(2):71-84.

12. Watanabe K, Matsumoto $M$, Tsuji $T$, et al. Ball tip technique for thoracic pedicle screw placement in patients with adolescent idiopathic scoliosis. J Neurosurg Spine 2010;13(2):246-52.

13. Obeid I, Bourghli A, Larrieu D, et al. The global tilt: evaluation of a parameter considering the global spinopelvic alignment. J Med Liban. 2016;64(3):146-51.

14. Obeid I, Boissiere L, Yilgor C, et al. Global tilt: a single parameter incorporating spinal and pelvic sagittal parameters and least affected by patient positioning. Eur Spine J. 2016;25(11):3644-9.

15. Qiao J, Zhu F, Xu L, et al. T1 pelvic angle: a new predictor for postoperative sagittal balance and clinical outcomes in adult scoliosis. Spine. 2014;39(25):2103-7.

16. Ryan DJ, Protopsaltis TS, Ames CP, et al. T1 pelvic angle (TPA) effectively evaluates sagittal deformity and assesses radiographical surgical outcomes longitudinally. Spine. 2014;39(15):1203-10.

17. Tokuhashi Y, Matsuzaki H, Oda H, et al. Clinical course and significance of the clear zone around the pedicle screws in the lumbar degenerative disease. Spine. 2008;33(8):903-8.

18. Sanden B, Olerud C, Petren-Mallmin M, et al. The significance of radiolucent zones surrounding pedicle screws. Definition of screw loosening in spinal instrumentation. J Bone Joint Surg Br. 2004;86 (3):457-61.

19. Fujiwara A, Kobayashi N, Saiki K, et al. Association of the Japanese Orthopaedic Association score with the Oswestry Disability Index, Roland-Morris Disability Questionnaire, and short-form 36. Spine. 2003;28(14):1601-7.

20. Ebata S, Ohba T, Oba H, et al. Bilateral dual iliac screws in spinal deformity correction surgery. J Orthop Surg Res. 2018;13(1):260.

21. Jacob HA, Kissling RO. The mobility of the sacroiliac joints in healthy volunteers between 20 and 50 years of age. Clin Biomech (Bristol, Avon). 1995;10(7):352-61.

22. Goode A, Hegedus EJ, Sizer P, et al. Three-dimensional movements of the sacroiliac joint: a systematic review of the literature and assessment of clinical utility. J Man Manip Ther. 2008;16(1): 25-38.

23. Vleeming A, Schuenke MD, Masi AT, et al. The sacroiliac joint: an overview of its anatomy, function and potential clinical implications. J Anat. 2012;221(6):537-67.

24. Weinberg DS, Morris WZ, Gebhart JJ, et al. Pelvic incidence: an anatomic investigation of 880 cadaveric specimens. Eur Spine J. 2016;25(11):3589-95.

25. Legaye J, Duval-Beaupere G, Hecquet J, et al. Pelvic incidence: a fundamental pelvic parameter for three-dimensional regulation of spinal sagittal curves. Eur Spine J. 1998;7(2):99-103.

26. Lee JH, $\mathrm{Na} \mathrm{KH}, \mathrm{Kim} \mathrm{JH}$, et al. Is pelvic incidence a constant, as everyone knows? Changes of pelvic incidence in surgically corrected adult sagittal deformity. Eur Spine J. 2016;25(11):3707-14.

27. Matteini LE, Kebaish KM, Volk WR, et al. An S-2 alar iliac pelvic fixation. Technical note. Neurosurg Focus. 2010;28(3):E13.

Spine Surgery and Related Research is an Open Access journal distributed under the Creative Commons Attribution-NonCommercial-NoDerivatives 4.0 International License. To view the details of this license, please visit (https://creativeco mmons.org/licenses/by-nc-nd/4.0/). 